\title{
Cardiac computed tomography reveals aortic valve perforation in a patient with severe aortic regurgi- tation
}

\author{
Namkyun $\mathrm{Kim}^{1}$, Sung Mok Kim²,3, Sung-Ji Park ${ }^{3,4}$, and Yeon Hyeon Choe ${ }^{2,3}$
}

${ }^{1}$ Cardiology Center, Kyungpook National University Chilgok Hospital, Daegu; ${ }^{2}$ Department of Radiology, ${ }^{3}$ Cardiovascular and Stroke Imaging Center, Heart Vascular Stroke Institute, ${ }^{4}$ Division of Cardiology, Department of Medicine, Samsung Medical Center, Sungkyunkwan University School of Medicine, Seoul, Korea
A 64-year-old man with moderate to severe aortic regurgitation without subjective symptoms who was followed for 7 months presented to the outpatient clinic with dyspnea. He had no history of fever or chills. C-reactive protein was elevated. Transthoracic echocardiography (TTE) revealed severe eccentric aortic regurgitation due to a prolapsed aortic valve (left coronary cusp and non-coronary cusp) and malcoaptation (Fig. 1A). These findings were worse than demonstrated on TTE 8 months prior (Fig. 1B). For

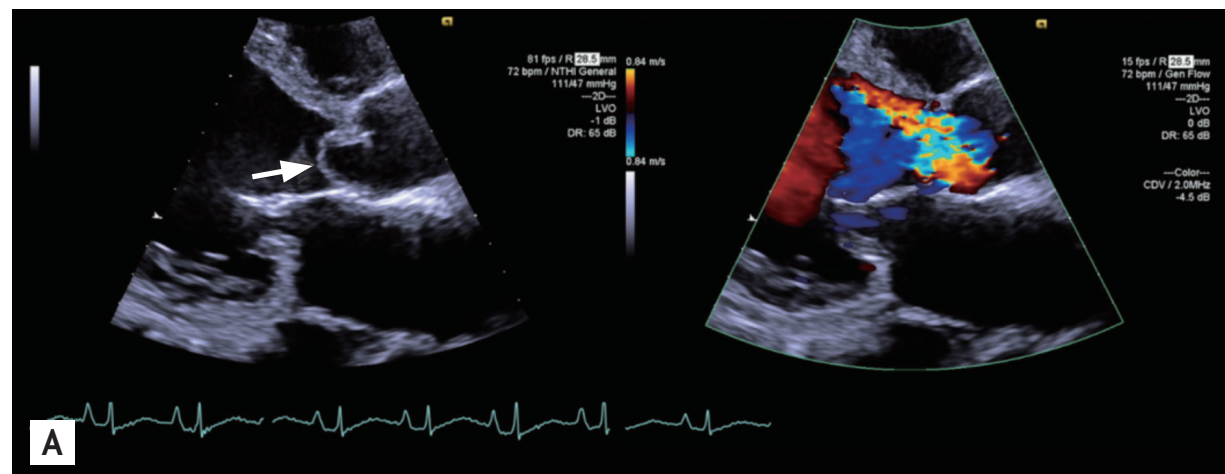

Received: March 17, 2018

Revised : May 28, 2018

Accepted: June 28, 2018

Correspondence to

Sung Mok Kim, M.D.

Tel: +82-2-3410-2519

Fax: +82-2-3410-2559

E-mail: sungmok_kim@hanmail.net
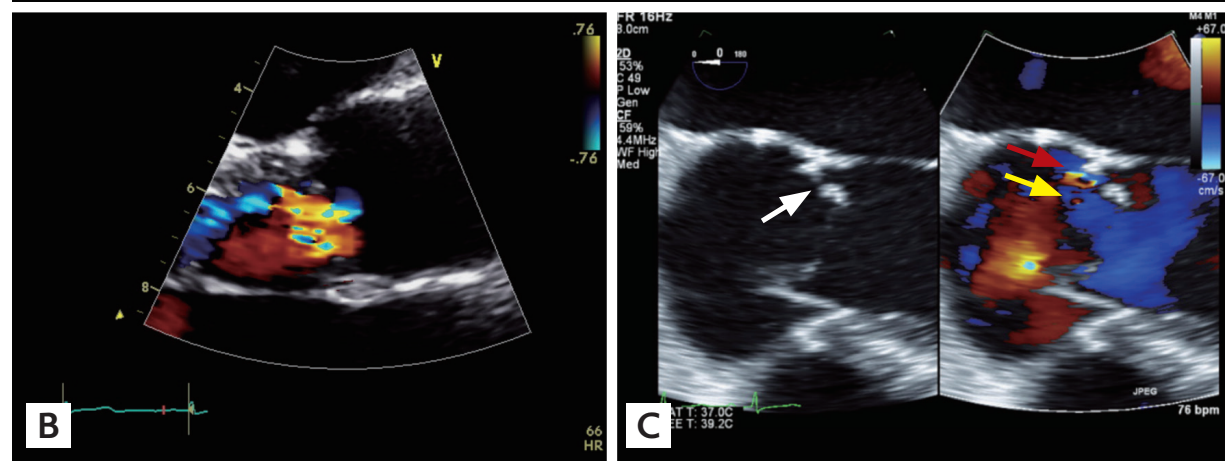

Figure 1. (A) Two-dimensional color Doppler transthoracic echocardiography shows a prolapsed non-coronary cusp of the aortic valve (white arrow) with severe eccentric aortic regurgitation on the parasternal long axis view. (B) Two-dimensional color Doppler transthoracic echocardiography performed 8 months before reveals less severe aortic regurgitation flow without a difference in direction. (C) Transesophageal echocardiography demonstrates a 2 -mm-sized perforation (white arrow) of the non-coronary cusp on the $0^{\circ}$ view biplane two-dimensional image. Color Doppler imaging shows two regurgitation flows, eccentric jets through a perforation site and through a prolapse site (red and yellow arrows). 


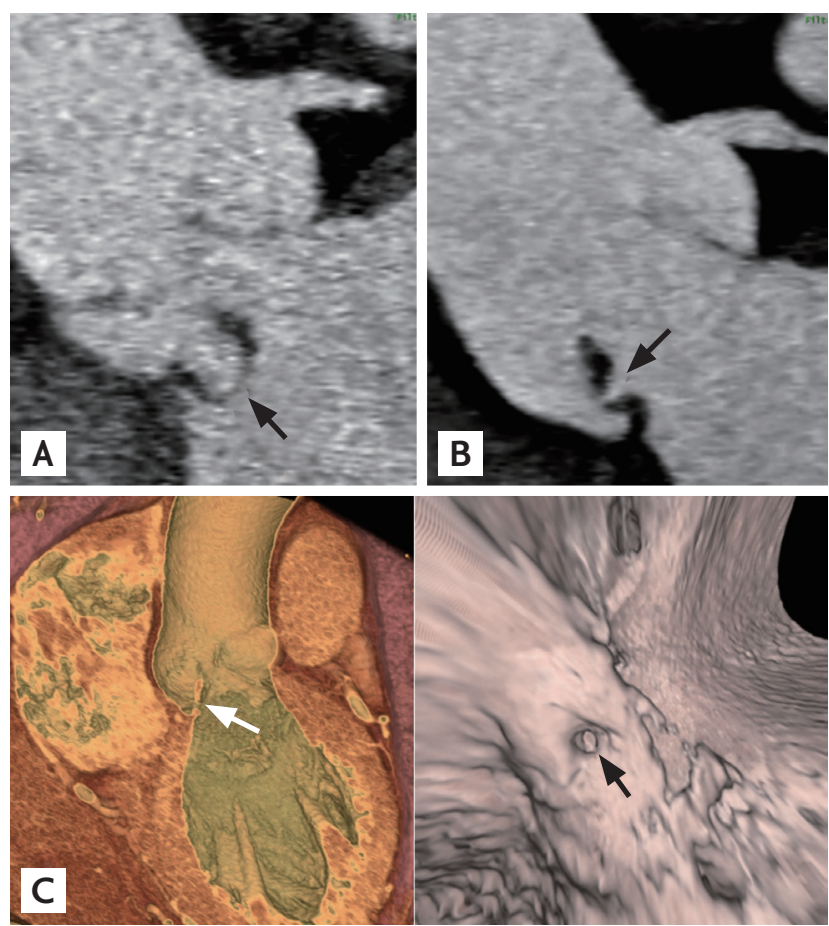

Figure 2. (A) Cardiac computed tomography demonstrates a prolapsed non-coronary cusp of the aortic valve (black arrow) during diastole on the cine image. (B) Aortic valve perforation of the non-coronary cusp is detected during systole in the cine image (black arrow). (C) Aortic valve perforation can be seen on the three-dimensional volume rendering and virtual endoscopic view (white and black arrows). the evaluation of coronary artery disease before elective aortic valve replacement, cardiac computed tomography (CT) was performed and demonstrated non-coronary cusp prolapse consistent with TTE as well as additional perforation (Fig. 2). Then, transesophageal echocardiography (TEE) confirmed the presence of a 2-mm-sized perforation in the non-coronary cusp (Fig. $1 \mathrm{C}$ ). In the surgical field, there was a deformity in the non-coronary cusp and right coronary cusp. After diagnosis with culture-negative endocarditis, the patient was treated with 4 weeks of ampicillin-sulbactam and 2 weeks of gentamicin. The patient recovered and was discharged uneventfully. We received informed consent from the patient. In this case, aortic valve perforation, which did not appear on TTE, was demonstrated on a three-dimensional reconstruction of electrocardiography-gated cardiac CT. The perforation was confirmed on TEE. Anatomical information regarding the coronary artery and great vessels obtained via CT could be useful for surgical planning.

\section{Conflict of interest}

No potential conflict of interest relevant to this article was reported. 